\title{
A CHARACTERIZATION OF HYPERBOLIC KATO SURFACES
}

\author{
MARCo Brunella ${ }^{\dagger}$
}

\begin{abstract}
We give a characterization of hyperbolic Kato surfaces in terms of the existence of an automorphic Green function on a cyclic covering. This is achieved by analysing a naturally defined Levi-flat foliation, and by perturbing certain Levi-flat leaves to strictly pseudoconvex hypersurfaces.
\end{abstract}

2010 Mathematics Subject Classification: Kato surfaces, Levi-flat foliations, plurisubharmonic functions.

Key words: 32J15, 32U05, 32V40.

\section{Introduction}

Kato surfaces are important examples of compact complex surfaces with first Betti number equal to 1, discovered by Kato more that 30 years ago [Kat], see also [Dlo] and [Nak, §5]. They occupy a central place in the current speculative theory of non-Kählerian surfaces. It is customary today to divide the class of Kato surfaces into three disjoint subclasses:

(1) Enoki surfaces $\left(\sigma_{n}=2 n\right.$ in the terminology of [Dlo]), which can be also described as exceptional compactifications of affine bundles over elliptic curves [Nak, $\S 4.5]$;

(2) Kato surfaces of intermediate type $\left(2 n<\sigma_{n}<3 n\right)$;

(3) Inoue-Hirzebruch surfaces $\left(\sigma_{n}=3 n\right)$, discovered by Inoue prior to Kato by a very different construction [Nak, $\S 4.1$ and $\S 4.3]$.

There is a fundamental difference between class (1) and classes (2) and (3), coming from the results of [D-O]. If $S$ belongs to class (2) or (3), then $S$ admits a Green function, i.e. a multiplicatively automorphic negative function on the universal covering $\widetilde{S}$ which is pluriharmonic outside an analytic subset $Z \subset \widetilde{S}$, along which the function has logarithmic

${ }^{\dagger}$ Note of the editors: this paper was submitted after Marco Brunella had passed away, following his family's will. We added four footnotes including referee's remarks. We thank Laurent Meersseman for his help. 
poles. Such a function, which is moreover unique up to a multiplicative constant, cannot exist on surfaces of class (1).

Due to this dichotomy, we shall refer to surfaces of class (2) or (3) as hyperbolic Kato surfaces, and to those of class (1) as parabolic Kato surfaces.

Our aim is to give a characterization of hyperbolic Kato surfaces in terms of existence of a Green function with nonempty polar set. This is analogous to the result of $[\mathbf{B r 2}]$ giving a characterization of Inoue surfaces (another important class of non-Kählerian surfaces, see [Nak, $\S 3])$ in terms of existence of a Green function without poles.

Theorem 1.1. Let $S$ be a compact connected complex surface of algebraic dimension zero. Suppose that there exists an infinite cyclic covering $\pi: \widetilde{S} \rightarrow S$ and a negative plurisubharmonic function $F: \widetilde{S} \rightarrow[-\infty, 0)$ such that:

(i) $F \circ \varphi=\lambda \cdot F$ for some positive $\lambda$, where $\varphi: \widetilde{S} \rightarrow \widetilde{S}$ is a generator of the group of deck transformations;

(ii) $d d^{c} F$ is supported on an analytic subset $Z \subset \widetilde{S}$;

(iii) $Z \neq \emptyset$.

Then $S$ is a (possibly blown up) Kato surface.

Even if this statement is formally close to $[\mathbf{B r 2}]$ (where $Z=\emptyset$ ), its proof follows a completely different path. The main idea is the following. The function $F$ induces on $S$ a (singular) codimension one real foliation $\mathcal{H}$, the leaves of which are noncompact Levi-flat hypersurfaces, spiralling around an arboreal cycle of rational curves. Using the nonemptyness of $Z$, we shall be able to "approximate" such a Levi-flat leaf by a compact strictly pseudoconvex hypersurface in $S$, not homologous to zero. Then the conclusion will be a consequence of [Br1].

The main technical point is therefore a result on the approximation of Levi-flat hypersurfaces with corners by smooth strictly pseudoconvex hypersurfaces, whose statement and proof will be given in the last part of the paper (Theorem 3.1), under a degree of generality going far beyond the context of Theorem 1.1.

\section{Proof of Theorem 1.1 modulo Theorem 3.1}

Let $S$ satisfy the assumptions of Theorem 1.1. As in [Br2, §1], we may also suppose that $S$ is minimal, and then Enriques-Kodaira classification leads to see that $S$ belongs to the class $\mathrm{VII}_{0}$, that is $b_{1}(S)=1$ and $\operatorname{kod}(S)=-\infty$. Moreover, $S$ cannot be a Hopf surface nor an Enoki surface: indeed, those surfaces contain a Zariski-open subset uniformised 
by $\mathbb{C}^{2}$, and this fact clearly prevents the existence of a nonconstant negative plurisubharmonic function on some covering (Liouville's theorem).

Denote by $\left\{Z_{j}\right\}_{j \in I}$ the irreducible components of $Z$, so that

$$
d d^{c} F=\sum_{j \in I} \mu_{j} \cdot \delta_{Z_{j}}
$$

for suitable positive real numbers $\left\{\mu_{j}\right\}_{j \in I}$. Here, as usual, $\delta_{Z_{j}}$ denotes the integration current on $Z_{j}$. The function $F$ is equal to $-\infty$ on $Z$ (logarithmic poles) and is finite and pluriharmonic on $\widetilde{S} \backslash Z$.

Since $F \circ \varphi=\lambda \cdot F$, the set $Z$ is $\varphi$-invariant, and so it projects to an analytic subset $C \subset S$. By results of Kodaira, Enoki and Nakamura [Nak, $\S 6$ and $\S 7$, each irreducible component of $C$ is a rational curve, and each connected component is either a tree of rational curves or a cycle of rational curves with possibly some trees attached (an arboreal cycle). Moreover, the intersection form of such a component is negative definite, so that the component is contractible to a normal singularity.

Lemma 2.1. Every connected component of $C$ is a (arboreal) cycle of rational curves.

Proof: Suppose, by contradiction, that $C_{0}$ is a connected component of tree type. Then $C_{0}$ is simply connected, and some neighbourhood $U$ of $C_{0}$ lifts isomorphically to $\widetilde{S}$. We obtain from $F$ a function $F_{0}: U \rightarrow$ $[-\infty, 0)$ such that $d d^{c} F_{0}$ is supported on $C_{0}$. This means that a nontrivial linear combination of the irreducible components of $C_{0}$ is $d d^{c}$-cohomologous to zero. But this contradicts the fact that the intersection form of $C_{0}$ is negative definite.

In particular, and since $C \neq \emptyset$, it follows from a result of Nakamura [Nak, $\S 8]$ that $S$ is a degeneration of blown up Hopf surfaces, i.e. there exists a family of surfaces over the $\operatorname{disc}\{S\}_{t \in \mathbb{D}}$ such that $S_{0}=S$ and $S_{t}$ is a blown up Hopf surface for every $t \neq 0$. This fact will allow later to use the result of $[\mathbf{B r} \mathbf{1}]$.

Still by Nakamura's results [Nak, $\S 7$ ] we may (and will) suppose that $C$ is connected, and that the inclusion of $C$ into $S$ induces an isomorphism between fundamental groups (otherwise $S$ is an Inoue-Hirzebruch surface and the proof is done).

Referee's note: this is obtained from a suitable version of the so-called "Federer Support Theorem", cf. Corollary 2.14, p. 143 in: J. P. Demailly, Complex Analytic and Differential Geometry, http://www-fourier.ujf-grenoble.fr/ demailly/ manuscripts/agbook.pdf. 
The function $\left.F\right|_{\widetilde{S} \backslash Z}$ induces a real analytic (singular) fibration

$$
f=\log (-F): S \backslash C \longrightarrow \mathbb{S}^{1}=\mathbb{R} /(\log \lambda) \cdot \mathbb{Z}
$$

The level sets of $f$ and the curve $C$ may be informally thought as a singular codimension one foliation $\mathcal{H}$ on $S$. A regular level set of $f$ is a leaf (or a union of leaves) which is properly embedded in $S \backslash C$ and accumulates to the full $C$, by spiralling around it.

Let us be more precise. Take an embedded real analytic circle $\gamma \subset$ $C$, not passing through the singularities (nodes) of $C$, such that $C \backslash$ $\gamma$ is connected. This is possible since $C$ is an arboreal cycle; thus $\gamma$ is contained in one of the irreducible components of $C$ which compose the cycle inside $C$, and in that component $\gamma$ separates the two nodes corresponding to the intersection with the other components of the cycle. Because $\gamma$ is homotopic to zero in $S$ (being contained in a rational curve), the function $F$ induces a function $F_{0}$ on a neighbourhood of $\gamma$, uniquely defined up to multiplication by $\lambda^{n}, n \in \mathbb{Z}$. This function $F_{0}$ has a logarithmic pole along $C$, and we can find holomorphic coordinates $(z, w)$ on a neighbourhood $V$ of $\gamma$ such that:

(1) $C \cap V=\{w=0\}$;

(2) $\gamma=\{w=0,|z|=1\}$;

(3) $F_{0}=\mu \cdot \log |w|+\beta \cdot \log |z|$, for some $\mu>0$ and $\beta \in \mathbb{R}$.

Here $z$ takes values in an annulus $\{1-\varepsilon<|z|<1+\varepsilon\}$ and $w$ in a disc $\{|w|<\varepsilon\}$, for some $\varepsilon>0$ (and $\varepsilon<1$, since $F_{0}$ is negative). The existence of those coordinates follows from the easy fact that a closed logarithmic 1-form with poles on $C$ can be put in the normal form $\mu \frac{d w}{w}+\beta \frac{d z}{z}$, around $\gamma$.

Let $\Gamma$ denote the solid torus embedded in $V$ corresponding to the points with $|z|=1$. Remark that it is a Levi-flat hypersurface, foliated by the holomorphic discs $\{z=c\}, c \in \gamma$.

Take now a regular level set of $f, W=W_{t}=f^{-1}(t)$. Since $F$ is pluriharmonic on $\widetilde{S} \backslash Z, W$ is a real analytic Levi-flat hypersurface, properly embedded in $S \backslash C$. It intersects $V$ along the level sets $F_{0}^{-1}\left(-\lambda^{n} e^{t}\right)$, $n \in \mathbb{Z}$, and $\Gamma$ along the countable collection of tori

$$
T_{n}=\left\{|z|=1,|w|=r_{n}\right\}, \quad n \geq 0,
$$

where $\left\{r_{n}\right\} \subset(0, \varepsilon)$ is a sequence decreasing to 0 (and, more explicitely, given recursively by $\log r_{n}=\lambda^{ \pm n} \cdot \log r_{0}$, with the sign \pm chosen depending on the sign of $\lambda-1$ ). 
Lemma 2.2. We have

$$
W \backslash \bigcup_{n \geq 0} T_{n}=\bigcup_{n \geq 0} \Omega_{n},
$$

where each $\Omega_{n}$ is open and relatively compact in $W$, and moreover:

(i) $\partial \Omega_{0}=T_{0}$;

(ii) $\Omega_{n}$ is connected and $\partial \Omega_{n}=T_{n}-T_{n-1}$ for every $n \geq 1$.

Proof: Let $\eta:[0,1] \rightarrow C$ be a closed path generating $\pi_{1}(C)$, starting and ending at some point of $\gamma$. It can be lifted to $W$, as a (nonclosed) path starting at some point of $T_{n}\left(n\right.$ large) and ending at some point of $T_{n+\ell}$ : this is just the holonomy of $\mathcal{H}$ along $\eta$, in a mildly singular context. Now, $\eta$ is also a generator of $\pi_{1}(S)$, and this means that if we prolonge $F_{0}$ along $\eta$ then, when we return to $V$, we get $\lambda^{ \pm 1} F_{0}$. In other words, we must have $\ell= \pm 1$. It follows that $T_{n}$ and $T_{n-1}$ bound a relatively compact domain $\Omega_{n} \subset W$, and $T_{0}$ bounds a relatively compact open subset $\Omega_{0} \subset W$.

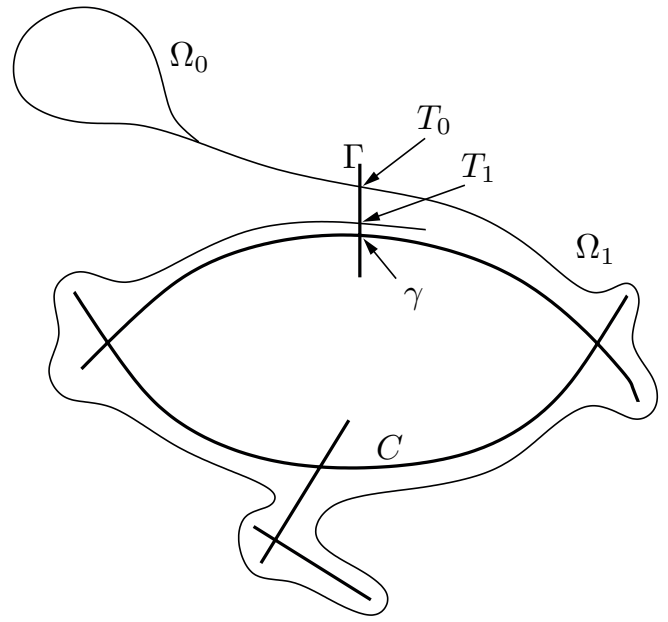

It is also easy to control the topology of the domains $\Omega_{n}, n \geq 1$ : each one is diffeomorphic to the boundary of a small tubular neighbourhood of $C$ from which the intersection with $\Gamma$ has been removed. This topology may be relatively complicated, because of the possible presence of the arborification of $C$ (if $C$ is just a cycle, without attached trees, then $\Omega_{n}$ is diffeomorphic to $\mathbb{T}^{2} \times(0,1)$, for every $\left.n \geq 1\right)$. Remark that we do not claim, in the previous lemma, that $\Omega_{0}$ is connected too: $W$ could have a compact connected component, far from $C$. But, anyway, 
$\Omega_{0}$ contains precisely one noncompact connected component, the one with boundary $T_{0}$, and this component will be denoted by $N$.

Consider now the Levi foliation on $W$, restricted to the compact connected threefold with boundary

$$
\bar{N}=N \cup T_{0} .
$$

Denote this foliation by $\mathcal{F}$, and note that it is transverse to the boundary, where it restricts to a linear foliation with slope $\beta / \mu$ (see (3) above).

Lemma 2.3. Every leaf of $\mathcal{F}$ intersects the boundary.

Proof: On a neighbourhood of $\bar{N}$ in $S$ the function $F$ induces a function $F_{1}$ (as usual, unique up to multiplication by a power of $\lambda$ ). The Levi foliation on $\bar{N}$ is then defined by the closed 1 -form $\omega=\left.d^{c} F_{1}\right|_{\bar{N}}$. It is then a standard fact that, depending on the periods of $\omega$, either $\mathcal{F}$ is a proper fibration over the circle, or every leaf of $\mathcal{F}$ is dense in $\bar{N}$. In both cases the conclusion is evident.

The torus $T_{0}$ is also boundary of a solid torus $B_{0} \subset \Gamma \subset S$. Take now

$$
M=N \cup T_{0} \cup B_{0} .
$$

It is a compact Levi-flat hypersurface with corners, which satisfies all the requirements of Theorem 3.1 of the next section. Hence, we deduce from that theorem that $M$ can be perturbed to a smooth strictly pseudoconvex hypersurface $\widetilde{M}$. Moreover, $\widetilde{M}$ is certainly not homologous to zero in $S$ : its intersection number with a generator of $\pi_{1}(C)$ (the loop $\eta$ of Lemma 2.2) is equal to \pm 1 . We can therefore apply the result of $[\mathbf{B r} \mathbf{1}]$ and conclude that $S$ is a Kato surface (of hyperbolic type, since we have already excluded the Enoki case).

\section{Levi-flat hypersurfaces with corners}

Here we switch to a more general context, and work on an arbitrary (not necessarily compact) complex surface $X$.

We shall say that a closed connected subset $M \subset X$ is a Levi-flat hypersurface with corners if for every $p \in M$ there exists a neighbourhood $U_{p}$ of $p$ in $X$, with coordinates $(z, w)$, such that $M \cap U_{p}$ is expressed either by

$$
\{\operatorname{Im} z=0\}
$$

Referee's note: the one whose closure has $T_{0}$ as boundary.

Referee's note: cf. Theorem 9.1.4, p. 206 in: A. Candel, L. Conlon, Foliations I, GSM vol. 23, Amer. Math. Soc., 2000. 
or by

$$
\{\operatorname{Im} z=0, \operatorname{Im} w \leq 0\} \cup\{\operatorname{Im} w=0, \operatorname{Im} z \leq 0\} .
$$

Thus, there is a smooth part $M^{0}$, where the first representation holds, and a singular part $M^{1}$. If $M_{j}^{1}$ is a connected component of $M^{1}$, then we have locally (on $M_{j}^{1}$ ) two smooth pieces of $M$ adjacent to $M_{j}^{1}$. To simplify the matter, we shall suppose that even globally we have two different connected components of $M^{0}$ adjacent to $M_{j}^{1}$ (instead of only one adjacent two times). Therefore, the closure $\overline{M_{k}^{0}}$ of a connected component $M_{k}^{0}$ of $M^{0}$ is an embedded connected threefold with boundary $\partial M_{k}^{0}=\cup_{j \in I_{k}} M_{j}^{1}$.

Every $\overline{M_{k}^{0}}$ is a Levi-flat hypersurface with boundary. We shall denote by $\mathcal{F}_{k}$ its Levi foliation; note that it is transverse to the boundary. Moreover, if $M_{j}^{1}$ is a commont component of $\partial M_{k}^{0}$ and $\partial M_{h}^{0}$, then the two boundary foliations $\left.\mathcal{F}_{k}\right|_{M_{j}^{1}}$ and $\left.\mathcal{F}_{h}\right|_{M_{j}^{1}}$ are mutually transverse.

Example 3.1. Take the standard totally real torus $T=\{|z|=|w|=$ 1\} $\subset \mathbb{C}^{2}$. Take two real analytic mutually transverse foliations $\mathcal{L}_{1}$ and $\mathcal{L}_{2}$ on $T$. These foliations can be complexified: there exists, on some neighbourhood $V$ of $T$, two holomorphic foliations $\mathcal{F}_{1}$ and $\mathcal{F}_{2}$ whose traces on $T$ are $\mathcal{L}_{1}$ and $\mathcal{L}_{2}$. Let now $M_{j}$ be the saturation of $T$ by $\mathcal{F}_{j}$, i.e. the union of the leaves of $\mathcal{F}_{j}$ intersecting $T$. Then, if $V$ is sufficiently small, $M_{1}$ and $M_{2}$ are real analytic Levi-flat hypersurfaces in $V$, intersecting transversely along $T$. By taking only "half" of $M_{1}$ and $M_{2}$ we get a Levi-flat hypersurface with corners in $V$.

Remark that a Levi-flat hypersurface with corners is, in particular, a topological manifold, and so the notion of orientability makes sense. If $M$ is orientable, then every connected component of $M^{1}$ is also orientable (being two-sided in $M$ ), and hence, when compact, it is a totally real torus. It is then not difficult to see that the previous example gives a universal local model for $M$ around such a component of $M^{1}$.

If $M$ is orientable and $U \subset X$ is a tubular neighbourhood, then $U \backslash M$ has two connected components. If $V_{j} \subset U$ is a tubular neighbourhood of $M_{j}^{1}$, then $V_{j} \backslash\left(M \cap V_{j}\right)$ has also two connected components, a "pseudoconvex" one $V_{j}^{+}$and a "pseudoconcave one" $V_{j}^{-}$: if $M$ is locally expressed by $\{\operatorname{Im} z=0, \operatorname{Im} w \leq 0\} \cup\{\operatorname{Im} w=0, \operatorname{Im} z \leq 0\}$ around a point of $M_{j}^{1}$, then $V_{j}^{+}$is the component containing $\{\operatorname{Im} z<0, \operatorname{Im} w<0\}$. We shall say that $M$ has a pseudoconvex side if all the $\left\{V_{j}^{+}\right\}$are contained in the same connected component of $U \backslash M$, which will henceforth be denoted by $U^{+}$(and the other by $U^{-}$). 
It is rather evident that if $M$ does not have a pseudoconvex side, then there is no hope for approximating $M$ by strictly pseudoconvex smooth hypersurfaces. In order to do such an approximation when a pseudoconvex side exists, we shall need an additional dynamical assumption, which will allow to propagate the strict pseudoconvexity concentrated on $M^{1}$ to the full $M$. This is somewhat related to the approximation of confoliations by contact structures $[\mathbf{E}-\mathbf{T}]$.

Theorem 3.1. Let $M \subset X$ be a compact, connected, orientable, Leviflat hypersurface with corners, admitting a pseudoconvex side. Suppose that for every smooth piece $\overline{M_{k}^{0}}$ the Levi foliation $\mathcal{F}_{k}$ has the following property: every leaf of $\mathcal{F}_{k}$ intersects the boundary $\partial M_{k}^{0}$. Then $M$ can be $C^{0}$-approximated by smooth strictly pseudoconvex hypersurfaces.

For the proof we shall need the following general fact.

Proposition 3.1. Let $N$ be a compact, connected, orientable manifold with boundary (of arbitrary dimension $n$ ), equipped with a smooth foliation by Riemann surfaces $\mathcal{F}$, transverse to the boundary and such that every leaf intersects the boundary. Then there exists a smooth function $\varphi$ on $N$ which is strictly subharmonic along the leaves of $\mathcal{F}$.

Proof: It is sufficient to prove the following: for every $p \in N$ there exists $\varphi \in C^{\infty}(N)$ such that $\Delta_{\mathcal{F}} \varphi \geq 0$ and $\left(\Delta_{\mathcal{F}} \varphi\right)(p)>0$. Here $\Delta_{\mathcal{F}}$ is the foliated laplacian with respect to a hermitian metric on the leaves.

Set $P=\{z \in \mathbb{C}|| z \mid<2, \operatorname{Re} z \geq 0\}, Q=\{z \in \mathbb{C}|| z \mid<1, \operatorname{Re} z \geq$ $0\}$. Since the leaf through $p$ intersects $\partial N$, we can find an embedding (foliated chart) $j: P \times \mathbb{R}^{n-2} \rightarrow N$, sending boundary to boundary, such that $j^{*}(\mathcal{F})$ is the horizontal foliation (with leaves $P \times\{t\}, t \in \mathbb{R}^{n-2}$ ) and $j\left(\frac{1}{2}, 0\right)=p$. We may also require that $\left.j\right|_{P \times\{t\}}$ is holomorphic for every $t$, with respect to the complex structure on the leaves of $\mathcal{F}$.

Take on $P$ a smooth function $\varphi_{0}$ such that:

(i) $\operatorname{Supp}\left(\varphi_{0}\right) \subset Q$;

(ii) $\Delta \varphi_{0} \geq 0$ on $P$;

(iii) $\left(\Delta \varphi_{0}\right)\left(\frac{1}{2}\right)>0$.

It is evident that such a function exists. Let $\chi \in C_{\mathrm{cpt}}^{\infty}\left(\mathbb{R}^{n-2}\right)$ be a nonnegative function with $\chi(0)>0$. Set $\varphi_{1}(z, t)=\chi(t) \cdot \varphi_{0}(z)$. Then $j_{*} \varphi_{1}$ can be extended, by zero, to the full $N$. The resulting $\varphi \in C^{\infty}(N)$ satisfies $\Delta_{\mathcal{F}} \varphi \geq 0$ and $\left(\Delta_{\mathcal{F}} \varphi\right)(p)>0$.

Remark 3.1. A variant of this construction produces $\varphi \in C^{\infty}(N \backslash \partial N)$ strictly subharmonic along the leaves and exhaustive $(\varphi(p) \rightarrow+\infty$ as $p$ tends to $\partial N)$. 
Let us now turn to the proof of Theorem 3.1.

Take a smooth piece $\overline{M_{k}^{0}}$ and a small tubular neighbourhood $U_{k} \subset X$ of it; we may assume that $\overline{M_{k}^{0}}$ prolonges to a real analytic hypersurface $\widetilde{M_{k}^{0}}$ (without boundary) properly embedded in $U_{k}$, so that $M_{k}^{0}$ is a relatively compact domain in $\widetilde{M_{k}^{0}}$. Up to shrinking $U_{k}$ around $\overline{M_{k}^{0}}$ (an operation that we will do again without further noticing), we dispose on $U_{k}$ of a strictly plurisubharmonic function: it is sufficient to take

$$
\psi=g_{0}^{2}+\varepsilon \varphi,
$$

where $g_{0}$ is any defining function of $\widetilde{M_{k}^{0}}, \varphi$ is the function provided by Proposition 3.1, smoothly extended to $U_{k}$ in any way, and $\varepsilon>0$ is sufficiently small. Indeed, at points of $\overline{M_{k}^{0}}$ we have $d d^{c} g_{0}=2 d g_{0} \wedge d^{c} g_{0}$, which is semipositive with Kernel $T^{\mathbb{C}} \frac{h}{M_{k}^{0}}$, and $d d^{c} \varphi$ is strictly positive on that Kernel.

Lemma 3.1. There exists a defining function $g$ of $\widetilde{M_{k}^{0}}$ such that $-\log |g|$ is strictly plurisubharmonic on $U_{k} \backslash \widetilde{M_{k}^{0}}$.

Proof: Start with the defining function $g_{0}$ already used before. Around a point of $\widetilde{M_{k}^{0}}$ choose coordinates $(z, w)$ such that $\widetilde{M_{k}^{0}}$ corresponds to $\{\operatorname{Im} z=0\}$, so that $g_{0}=e^{-h} \cdot \operatorname{Im} z$ for some $h$ smooth. Then $d d^{c}\left(-\log \left|g_{0}\right|\right)=$ $d d^{c} h+d d^{c}(-\log |\operatorname{Im} z|)$ admits a lower bound outside $\operatorname{Im} z=0$, since the first term is bounded and the second one is positive. Thus, by compactness of $\overline{M_{k}^{0}}$, there exists a (possibly negative) constant $C$ such that $d d^{c}\left(-\log \left|g_{0}\right|\right)>C \cdot d d^{c} \psi$ on $U_{k} \backslash \widetilde{M_{k}^{0}}$. It is then sufficient to take $g=e^{-|C| \cdot \psi} \cdot g_{0}$.

The level sets

$$
\widetilde{M_{k, \varepsilon}^{0}}=\{g=\varepsilon\}, \quad \varepsilon \neq 0
$$

are therefore smooth strictly pseudoconvex hypersurfaces converging to $\widetilde{M_{k}^{0}}$ as $\varepsilon \rightarrow 0$, from both sides.

Remark 3.2. The domains $\{|g|<\varepsilon\}, \varepsilon>0$, constitute a basis of strictly pseudoconcave neighbourhoods of $\widetilde{M_{k}^{0}}$. As observed in [F-L], the existence of a basis of strictly pseudoconvex neighbourhoods is a more subtle problem, involving for instance the holonomy of the foliation.

We apply Lemma 3.1 to every piece $\overline{M_{k}^{0}}$ of $M$, getting strictly pseudoconvex hypersurfaces $\widetilde{M_{k, \varepsilon}^{0}}$ in the pseudoconvex side of $M$.

Referee's note: $d d^{c}\left(g_{0}^{2}\right)=2 d g_{0} \wedge d^{c} g_{0}$. 


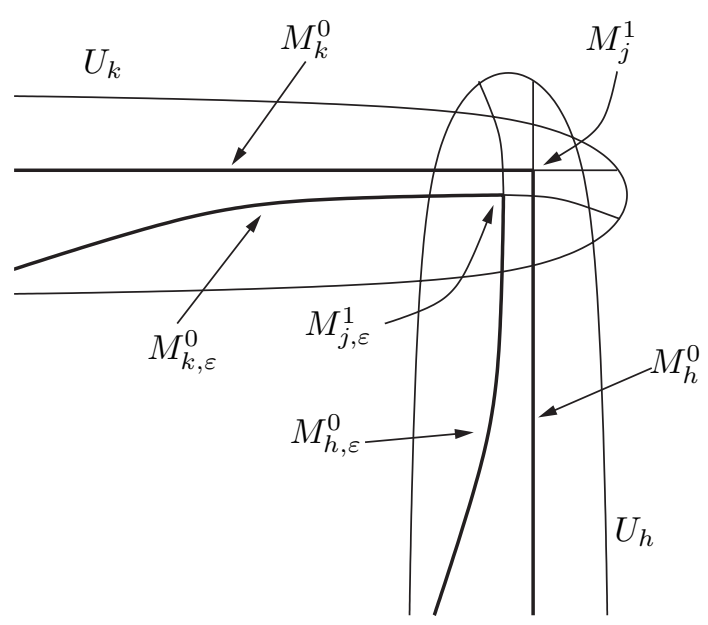

If $\widetilde{M_{k}^{0}}$ and $\widetilde{M_{h}^{0}}$ intersect along some torus $M_{j}^{1}$, then $\widetilde{M_{k, \varepsilon}^{0}}$ and $\widetilde{M_{h, \varepsilon}^{0}}$ intersect along some still totally real torus $M_{j, \varepsilon}^{1}$. We define $M_{k, \varepsilon}^{0} \subset \widetilde{M_{k, \varepsilon}^{0}}$ as the relatively compact domain bounded by those tori, so that the subset

$$
M_{\varepsilon}=\left(\bigcup_{k} M_{k, \varepsilon}^{0}\right) \cup\left(\bigcup_{j} M_{j, \varepsilon}^{1}\right)
$$

is a topological manifold with corners close to $M$, strictly pseudoconvex in its smooth part.

It remains to smooth the corners of $M_{\varepsilon}$. To do so, observe that in some neighbourhood $W$ of $M_{j, \varepsilon}^{1}$ the hypersurface $M_{\varepsilon}$ can be expressed by

$$
\left\{\operatorname{Max}\left(g_{k}, g_{h}\right)=0\right\}
$$

where $g_{k}$ and $g_{h}$ are smooth strictly plurisubharmonic functions defining $\widetilde{M_{k, \varepsilon}^{0}}$ and $\widetilde{M_{h, \varepsilon}^{0}}$. Let $\operatorname{Max}_{\delta}: \mathbb{R}^{2} \rightarrow \mathbb{R}$ be a regularized maximum function (i.e. a smooth convex function equal to Max outside an euclidean $\delta$-neighbourhood of the diagonal). Then $\operatorname{Max}_{\delta}\left(g_{k}, g_{h}\right)$ is smooth and strictly plurisubharmonic, and for $\delta$ sufficiently small its zero set is a smooth and strictly pseudoconvex hypersurface in $W$, close to $M_{\varepsilon} \cap W$ and equal to it ouside a compact subset of $W$. It is sufficient now to replace $M_{\varepsilon} \cap W$ with that smooth hypersurface. 


\section{References}

[Br1] M. Brunella, Un complément à l'article de Dloussky sur le colmatage des surfaces holomorphes, Ann. Inst. Fourier (Grenoble) 58(5) (2008), 1723-1732. DOI: 10.5802/aif. 2396.

[Br2] M. Brunella, A characterization of Inoue surfaces, Comment. Math. Helv. 88(4) (2013), 859-874. DOI: 10.4171/CMH/305.

[Dlo] G. Dloussky, Structure des surfaces de Kato, Mém. Soc. Math. France (N.S.) 14 (1984), 120 pp.

[D-O] G. Dloussky and K. Oeljeklaus, Vector fields and foliations on compact surfaces of class $\mathrm{VII}_{0}$, Ann. Inst. Fourier (Grenoble) 49(5) (1999), 1503-1545.

[E-T] Y. M. Eliashberg and W. P. Thurston, "Confoliations", University Lecture Series 13, American Mathematical Society, Providence, RI, 1998.

[F-L] F. Forstnerič and C. Laurent-ThiÉBaut, Stein compacts in Levi-flat hypersurfaces, Trans. Amer. Math. Soc. 360(1) (2008), 307-329 (electronic). DOI : 10.1090/S0002-9947-07-04263-8.

[Kat] Ma. Kato, Compact complex manifolds containing "global" spherical shells. I, in: "Proceedings of the International Symposium on Algebraic Geometry" (Kyoto Univ., Kyoto, 1977), Kinokuniya Book Store, Tokyo, 1978, pp. 45-84.

[Nak] I. NAKAmURA, Classification of non-Kähler complex surfaces, (Japanese), Sügaku 36(2) (1984), 110-124; Translated in Sugaku Expositions 2(2) (1989), 209-229.

Institut de Mathématiques de Bourgogne

UMR 5584

9 Avenue Savary

21078 Dijon

France

Primera versió rebuda el 8 de març de 2013, darrera versió rebuda el 18 de juliol de 2013. 